\title{
The role of gender inequalities in adolescent depression
}

The gender difference in the prevalence of depression is one of the most robust findings in psychiatric epidemiology and has been replicated across many cultures. ${ }^{1}$ Women are twice as likely to experience depression compared with men. ${ }^{1}$ This gender difference is the result of a sharp increase in the incidence of depression in girls during mid-adolescence. ${ }^{2}$

There are many theories for the gender difference in depression, several of which are likely to be important. ${ }^{3}$ Explanations can be divided into two broad categories, internal and external. Internal factors refer to biological or psychological characteristics, such as sex hormones or differences in cognitive vulnerability. External factors, in contrast, are environmental or societal, such as child sexual abuse. However, the distinction between internal and external factors is an artificial one. The external environment in which people develop also influences their own vulnerability, thus becoming internalised. Gender inequalities occur from birth onwards, and could lead to increased vulnerabilities to depression in girls.

According to cognitive models of depression, an individual's early experiences can lead to negative self-concepts that influence how they perceive, interpret, and remember our environment, and can increase the risk of depression. ${ }^{4}$ We propose that gender should be classified as an exposure variable within this causal pathway. For example, being female might lead to a more negative environment during childhood. This environment could lead to more negative cognitions, which increase the risk of depression. By adolescence, girls might have learnt to have more negative attitudes towards themselves because of how they are represented and treated in society. Societal inequalities during development could therefore directly influence negative self-concepts, lead to negative biases in information processing, and contribute to the increased incidence of depression in girls during adolescence.

Gender inequalities start early in life, are pervasive, and exist in most societies. Boys and girls are often treated differently from birth, and frequently have different behaviour expected from each of them. Many societies have norms that assign higher value to being a man than being a woman. ${ }^{5}$ Men and boys are often expected to fulfil masculine stereotypes of confidence and bravery, whereas many traditional and stereotypical characteristics of women and girls (passivity, emotionality, helplessness) are similar to common symptoms of depression. ${ }^{5}$ On average, men have consistently higher self-esteem than women. ${ }^{6}$ By early adolescence, boys take more risks than girls and gender differences in risk-taking during adolescence are smaller in countries with higher gender equality. ${ }^{7}$ Across Europe, higher national gender equality is related to a lower prevalence of depression and a smaller gender difference in depression for some groups. ${ }^{8}$

However, few studies have investigated whether gender inequalities lead to negative biases in information processing. An important approach to answering this question is to test whether girls demonstrate more negative biases on cognitive tasks, which provide a behavioural measure of 
automatic information processing. Gender differences in cognitive tasks measuring negative biases could be interpreted as being a result of societal and gender inequality. If gender leads to experiencing inequality which leads to negative cognitions, studies could test whether negative information processing biases mediate the association between gender and depression.

Cognitive vulnerability from information processing biases might be a way to address the emergence of depressive symptoms in adolescence. Changing gender norms and reducing gender inequality is challenging and progress is slow. Interventions that could directly reduce negative biases in information processing might increase resilience to stressors and reduce depressive symptoms in girls and boys. Public health strategies could also target more basic information processing in order to successfully change behaviour and prevent health problems. ${ }^{9}$

Young women (aged 16-24 years) were recognised as a group at high risk of common mental disorders, such as depression, in the 2014 UK Psychiatric Morbidity Survey. ${ }^{10}$ Despite the apparent improvements in gender norms in developed societies, with more people championing gender equality, the prevalence of depression is increasing more in young women than young men in the UK. ${ }^{10}$ The \#MeToo movement has highlighted that some of the perceived improvements in the position of women and girls in high income countries might be rather superficial.

Identifying modifiable causes of the gender difference in depression would have enormous health benefits but is still a neglected research priority. There is a crucial need for efforts at both a population and individual level to reduce the effect of gender inequality on health.

\section{Authors}

*Jessica K Bone ${ }^{1}$, Gemma Lewis ${ }^{1}$, Glyn Lewis ${ }^{1}$

${ }^{1}$ Division of Psychiatry, Faculty of Brain Sciences, University College London, UK.

*jessica.bone.15@ucl.ac.uk

\section{Conflicts of interest}

JKB reports a PhD studentship from the Economic and Social Research Council, during the conduct of the study. GeL and GIL report grants to University College London. We declare no competing interests.

\section{References}

1 Salk RH, Hyde JS, Abramson LY. Gender Differences in Depression in Representative National Samples: Meta-Analyses of Diagnoses and Symptoms. Psychol Bull 2017; 143: 783-822.

2 Hankin BL, Abramson LY, Moffitt TE, Silva PA, McGee R, Angell KE. Development of depression from preadolescence to young adulthood: emerging gender differences in a 10year longitudinal study. J Abnorm Psychol 1998; 107: 128-140.

$3 \quad$ Kuehner $\mathrm{C}$. Why is depression more common among women than among men? The Lancet Psychiatry 2017; 4: 146-58.

4 Beck AT. The evolution of the cognitive model of depression and its neurobiological correlates. Am J Psychiatry 2008; 165: 969-77. 
5 Chandra-Mouli V, Plesons $\mathrm{M}, \mathrm{Amin} \mathrm{A}$. Addressing harmful and unequal gender norms in early adolescence. Nat Hum Behav 2018; 2: 239-40.

6 Bleidorn W, Arslan RC, Denissen JJA, et al. Age and Gender Differences in Self-Esteem - A Cross-Cultural Window. J Pers Soc Psychol 2016; 111: 396-410.

7 Cárdenas JC, Dreber A, von Essen E, Ranehill E. Gender differences in competitiveness and risk taking: Comparing children in Colombia and Sweden. J Econ Behav Organ 2012; 83: 1123.

8 van de Velde S, Huijts T, Bracke P, Bambra C. Macro-level gender equality and depression in men and women in Europe. Sociol Heal IIIn 2013; 35: 682-98.

9 Marteau TM, Hollands GJ, Fletcher PC. Changing Human Behavior to Prevent Disease: The Importance of Targeting Automatic Processes. Science 2012; 337: 1492-5.

10 McManus S, Bebbington P, Jenkins R, Brugha T (eds.). Mental Health and Wellbeing in England: Adult Psychiatric Morbidity Survey 2014. Leeds: NHS Digital, 2016. 